\title{
Les pèlerinages de l'Afrique francophone subsaharienne en Terre Sainte : la formation discursive d'une communauté de croyants
}

The Francophone sub-Saharan Africa Pilgrimages to the Holy Land: The Discursive Formation of a Community of Believers

\section{Tal Sela}

\section{OpenEdition Journals}

Electronic version

URL: https://journals.openedition.org/aad/5428

DOI: $10.4000 /$ aad. 5428

ISSN: 1565-8961

Publisher

Université de Tel-Aviv

\section{Electronic reference}

Tal Sela, "Les pèlerinages de l'Afrique francophone subsaharienne en Terre Sainte : la formation

discursive d'une communauté de croyants", Argumentation et Analyse du Discours [Online], 27 | 2021

Online since 14 October 2021, connection on 11 November 2021. URL: http://journals.openedition.org/ aad/5428; DOI: https://doi.org/10.4000/aad.5428

This text was automatically generated on 11 November 2021 .

\section{cc)}

Argumentation \& analyse du discours est mis à disposition selon les termes de la licence Creative Commons Attribution - Pas d'Utilisation Commerciale - Pas de Modification 4.0 International. 


\section{Les pèlerinages de l'Afrique francophone subsaharienne en Terre Sainte : la formation discursive d'une communauté de croyants}

The Francophone sub-Saharan Africa Pilgrimages to the Holy Land: The Discursive Formation of a Community of Believers

Tal Sela

\section{Introduction}

1 Cet article se propose d'analyser les discours du pèlerinage en Terre Sainte depuis l'Afrique occidentale francophone ${ }^{1}$. Il explore un corpus constitutif de l'imaginaire du pèlerinage en mettant en évidence le rôle discursif et argumentatif du couple notionnel que constituent le singulier (le pèlerin isolé) et le collectif (son groupe de référence et d'appartenance identitaire).

2 Le pèlerinage est, dans les termes d'Alan Morinis (1992:4), « un voyage entrepris par une personne en quête d'un lieu ou d'un état qu'elle croit incarner un idéal précieux »: le fidèle part à la recherche de Dieu dans des lieux considérés comme sacrés. Il s'y rend pour fortifier sa foi, exalter sa ferveur, assister à des miracles, obtenir le pardon pour des fautes morales, solliciter une guérison, traverser une expérience spirituelle de profonde transformation, accomplir des obligations religieuses ou des vœux, ou simplement pour visiter des sites d'importance historique pour sa propre religion. Le pèlerinage, ainsi défini, est centré sur le désir de salut du pèlerin, sur sa quête d'une relation avec la divinité (Ron et Timothy 2019, Tomasi 2002 et al.). Ce cadre 
définitionnel renvoie le voyage de pèlerins aux motivations personnelles qui poussent le pèlerin à prendre la route.

3 Mais derrière ces motivations patentes, avouées par les pèlerins eux-mêmes, se profile une dimension latente, liée au phénomène de groupe et à son emprise sur le pèlerin isolé. Turner (1973) et Turner et Turner (1978) ont conceptualisé la dimension collective du pèlerinage par la célèbre notion de communitas. Leur recherche s'intéresse aux voyageurs religieux réunis en communauté de conviction, où les questions sociales de la vie quotidienne sont écartées de l'expérience spirituelle qu'ils traversent durant leur visite dans les sites de cultes. Lorsque les pèlerins se soustraient aux sollicitations du monde extérieur et à leurs préoccupations de tous les jours, ils se livrent plus facilement à la méditation intérieure et à l'introspection. Une communitas est ainsi formée en cours de route par des pèlerins qui cultivent ensemble une affinité de foi, et celle-ci transforme le voyage en expérience sacrée.

4 Le travail précurseur de Turner a défriché le terrain et ouvert la voie à de nombreuses recherches. Ces travaux montrent comment le pèlerinage a, depuis plusieurs siècles, promu la dimension groupale en en faisant un facteur crucial de l'expérience religieuse individuelle, et non pas en l'opposant à celle-ci. Dès lors, il n'est plus possible d'enfermer le pèlerin - figure mobile, traversant des paysages, des ethnies, des images et des histoires - dans la seule vocation religieuse qu'il cherche à accomplir. Celle-ci doit être observée à l'aune de toute une gamme de possibilités contemporaines offertes aux pèlerins pour forger par exemple de nouveaux liens communautaires, questionner et/ou confirmer les traditions religieuses, engendrer de nouvelles façons d'imaginer l'avenir et de le transformer au niveau individuel et collectif (Feldman 2017 : 79).

5 Cette étude de la dimension individuelle et collective du pèlerinage en Terre Sainte depuis l'Afrique occidentale francophone suscite plus encore l'intérêt quand on voit, ces dernières années, la montée en puissance des Églises évangéliques et pentecôtistes issues de la matrice chrétienne protestante, et la passion philo-sémitique pour Israël qui anime ces Églises. Cette passion s'enracine dans une théologie dite du Christianisme sioniste. Cette dernière émerge comme une force politique vers la fin du $19^{\mathrm{e}}$ siècle, considérant la construction d'un foyer national pour le peuple juif comme l'accomplissement de la prophétie biblique (Merkley 1998). Cette mouvance religieuse et politique gagne du terrain aux États-Unis par le biais du courant pentecôtiste (Stockton 1995, Fath 2005) et se propage dans le monde entier (Lacoste 2005). Si les Églises nord-américaines investissent des fonds considérables pour diffuser la Bonne Nouvelle sur les cinq continents, il faut savoir que la plupart des grands mouvements évangéliques évoluent en Afrique depuis des décennies durant les périodes pré- et postindépendance, indépendamment de toute influence américaine (Dorier-Apprill et Ziavoula 2005). Qu'il soit autonome ou sous tutelle américaine, le réseau évangélique fait partie d'une communauté discursive qui s'accroît à l'échelle mondiale, avec des répercussions importantes sur la structure sociale de nombreux pays.

6 La présence et l'influence de la mouvance évangélique à l'échelle locale ont été déjà étudiées dans le contexte de la Côte d'Ivoire (Raynal 2005, Miran-Guyon 2014), du Bénin (Cédric Mayrargue 2014), ainsi que de l'Afrique centrale (Dorier-Apprill et Ziavoula 2005). Ces recherches ignorent cependant la représentation religieuse d'Israël dans ces communautés. Cette représentation est évoquée dans un blog intitulé "Afrique subsaharienne et sionisme évangélique $»^{2}$, publié sur le site de Sciences- $\mathrm{Po}^{3}$, où son auteur, Sébastien Fath, présente quelques aspects de la judéophilie qui caractérisent le 
protestantisme évangélique subsaharien. ${ }^{4}$ Fath s'appuie sur une annonce du journal électronique abidjan.net (du 3 décembre 2011) pour évoquer l'initiative prise par l'État ivoirien d'envoyer 200 pèlerins en Israël. S'il évoque le cas précis du voyage de pèlerins en provenance de Côte d'Ivoire, notre recherche voudrait, quant à elle, explorer plus largement la dimension culturelle - discursive et argumentative - du pèlerinage effectué à partir de l'Afrique francophone subsaharienne, en s'intéressant plus particulièrement aux rapports d'interdépendance qui se nouent entre le pèlerin africain et le groupe qui l'accompagne.

7 Le présent article se propose donc de montrer comment cette relation d'interdépendance se tisse à même les textes verbaux et visuels qui accompagnent le voyage de pèlerins, depuis sa conception dans le pays d'origine jusqu'au retour au pays, suite au voyage. L'analyse discursive et argumentative d'une publicité télévisuelle, promouvant le pèlerinage en Terre Sainte, diffusée depuis Abidjan (Côte d'Ivoire) sera l'objet de la première partie de cet article. La deuxième partie sera consacrée à l'analyse du discours et de l'argumentation des brochures publicitaires pour les voyages organisés par l'agence de voyage Gota, située à Cotonou (Bénin). Cette analyse aura recours aux outils de la sémiotique sollicités par l'étude de l'image publicitaire et les liens qu'elle entretient avec le texte qui l'accompagne. L'analyse des attestations et les décorations que les pèlerins obtiennent durant le séjour en Israël et l'importance qu'ils acquièrent de retour au pays seront l'objet de la troisième partie.

\section{Prélude à un voyage de pèlerins (I) : LMTV et l'Ambassade chrétienne internationale de Jérusalem}

8 La télévision chrétienne Lumière du monde (LMTV) a été fondée en 2008 par son directeur M. Dieudonné Gollet ${ }^{5}$. Interviewé dans le cadre de cette recherche, Gollet exprime un très fort attachement pour Israël et une adhésion explicite au sionisme. Le directeur veut que la chaîne soit « un canal pour mieux connaître Israël ». Et pour ce faire, il entend consacrer deux heures d'émission par jour sur la société et l'actualité israélienne ${ }^{6}$. LMTV, dit Gollet, se présente comme « le bras d'Israël en Afrique ». Gollet prend l'initiative d'emmener des Africains en Terre Sainte suite à sa visite en Israël en 2017. Un an après, la chaîne réussit à regrouper 300 pèlerins venus de différents pays de l'Afrique occidentale francophone pour fêter en Terre Sainte les 70 ans de l'État d'Israël. La chaîne joue un rôle coordinateur reliant les pasteurs dans les communautés locales à l'agence de voyage qui s'occupe aussi bien du séjour en Terre Sainte que de la préparation administrative avant le départ (distribution des visas et autres autorisations).

LMTV organise ses voyages avec le concours de Sar-El tours, une agence de voyage israélienne spécialisée, depuis 1993, dans le domaine du "tourisme chrétien évangélique vers Israël $\aleph^{7}$. Les liens tissés avec des spécialistes locaux sont indispensables pour la réussite des voyages; encore faut-il les promouvoir parmi des pèlerins potentiels dans les pays d'origine. LMTV diffuse sur sa chaîne la promotion du voyage en Terre Sainte. Elle dit vouloir répondre au besoin de milliers d'Africains, qui, selon Gollet, « veulent venir en Israël mais ne savent pas comment le faire. » En 2019, la tâche de renforcer le message publicitaire est confiée aux mains de Jürgen Bühler, le président de l'Ambassade Chrétienne internationale, basée à Jérusalem (ICEJ), organisation évangélique ancrée dans le sillage du christianisme sioniste ${ }^{8}$. 

la chaîne LMTV, pour inviter les téléspectateurs à la fête des tabernacles, organisée à Jérusalem par l'Ambassade Chrétienne de Jérusalem durant le mois d'octobre 2019. Les deux vidéos suivent le même script à quelques différences près. Une vidéo débute par le cri feast ("fête ») prononcé par des groupes de participants munis de drapeaux de leur pays d'origine. Il est suivi par le discours de Bühler, en costume et en cravate, sur le fond du bandeau publicitaire de la chaine.

11 Son discours est accompagné d'images de la fête qui s'est déroulée lors d'une année précédente - on peut y voir les participants dans une salle de concert brandir des drapeaux nationaux, chanter et prier avec grande dévotion, sur le fond d'une musique rythmée. D'autres images sont prises dans les rues de Jérusalem ou dans d'autres lieux de cultes. L'identité nationale des pèlerins est mise en valeur par les drapeaux, comme pour marquer la diversité humaine et attester de la réalisation de la promesse biblique évoquée dans le discours que le président profère dans le cadre du film.

Le premier énoncé renvoie à l'ethos préalable du locuteur.

[1] Bonjour, je m'appelle Jürgen Bühler, je suis le Président de l'Ambassade Chrétienne de Jérusalem. Je suis là en Côte d'Ivoire dans les studios de LMTV. Et je suis là pour vous inviter personnellement à la fête des tabernacles à Jérusalem.

Le locuteur se présente en tant que "Président de l'Ambassade Chrétienne de Jérusalem ». Ce titre confère au discours une légitimité que le locuteur fonde sur son autorité institutionnelle de « diplomate » - notion associée au registre du discours de la politique internationale - et d'homme de la foi chrétienne. Cette autorité repose également sur la valeur doxique du nom iconique de la ville sainte de Jérusalem, dont il est le représentant. L'énoncé « Je suis là en Côte d'Ivoire dans les studios de LMTV... » établit la scénographie requise pour s'adresser à l'auditoire. Les studios de LMTV, chaîne de télévision ayant pour but de diffuser le message de Dieu partout en Afrique, sont un lieu privilégié pour valider la parole du locuteur. Le déictique «Je suis là en Côte d'Ivoire » a pour effet d'attirer l'attention des spectateurs sur un locuteur venu de loin et qui les honore de sa présence physique ; la distance parcourue depuis Jérusalem est constitutive d'un ethos d'apôtre qui s'affirme progressivement tout au long du discours. Cette scénographie implique aussi l'engagement attendu du public, basé sur le principe de la réciprocité et du respect pour un homme de Foi : si je suis venu vous voir en Côte d'Ivoire, vous êtes capables, à mon instar, de venir me rejoindre à Jérusalem.

L'identité des partenaires de l'énonciation - la figure de l'énonciateur-apôtre s'adressant à un public de spectateurs-croyants, va de pair avec la définition d'un lieu le plateau de la télé chrétienne - et d'un moment d'énonciation - la veille de la fête des tabernacles. Cette scénographie à partir de laquelle se développe le discours lui permet de fonder sa légitimité en vue d'agir sur le destinataire à la lumière d'une action bien déterminée (Maingueneau 2002 : 517) :

[2] La fête des tabernacles est une célébration et le prophète Zacharie au chapitre 14 déclare que toutes les nations du monde viendront à Jérusalem pour adorer le Roi, le Seigneur des seigneurs dans la ville du Grand roi.

La référence biblique opère la transmutation du discours en sermon télévisé. Dans un numéro de la revue Langage et société, Dominique Maingueneau analyse la manière dont le prédicateur du sermon montre « dans son énonciation même qu'il est contraint, par une nécessité intérieure, de parler comme il le fait » (2009). En parlant, le prédicateur s'inscrit dans le dispositif du sermon chrétien où il revêt le rôle de médiateur. Autorisé 
de la parole de Dieu, le prédicateur s'entremet entre les croyants, en quête perpétuelle d'un salut, et l'univers céleste de Dieu, garant du rapport établi par le sermon entre les participants de la prédication et l'institution cléricale qui les assemble (Maingueneau 2009 : 44). La reproduction de l'isotopie du sermon renforce le programme narratif dont la visée est d'inciter les fidèles à entreprendre un pèlerinage ${ }^{9}$.

En effet, la prophétie proférée par Zacharie est aussi bien une légitimation qu'une vocation que le prédicateur se sent obligé de suivre, tout en adressant au public l'obligation divine de participer à sa réalisation. C'est un ethos "inspiré " (Maingueneau 2009) qui se construit dans le discours et donne l'impression qu'une voix transcendante, celle du prophète Zacharie, parle à travers sa voix.

[3] L'opportunité vous est donnée de participer à la fête pour accomplir une prophétie biblique mais aussi de voir Dieu à l'œuvre sur la terre d'Israël. [4]

L'énoncé [3] a pour visée d'expliciter aux futurs pèlerins leur tâche («accomplir une prophétie biblique »). Il ne s'agit plus d'une invitation (" je suis là pour vous inviter... ») que l'ambassadeur de Jérusalem adresse aux spectateurs d'Afrique, mais d'une mission qu'il leur demande d'accomplir en les enjoignant à se fier au décret divin. L'énoncé "L'opportunité vous est donnée " accroît la pression exercée sur les fidèles: il transforme le moment de l'énonciation en une occasion rare qu'il ne faut pas manquer. Le prédicateur fait la promesse à ses spectateurs-paroissiens de "voir Dieu à l'œuvre sur la terre d'Israël», et de le rencontrer («Dieu vous révélera ses promesses pour vous»), acte de langage qui le transforme en prophète en inscrivant son énonciation dans les temps bibliques des miracles et des révélations divines. Cet effet discursif est renforcé davantage encore par le verbe de perception "voir", qui exprime une focalisation à travers le pèlerin. L'effet de point de vue (Rabatel 1998) contourne le topos religieux selon lequel « il faut croire sans voir ». Un homme pieux doit se contenter de voir Dieu par l'esprit, alors qu'il aimerait logiquement le voir de ses propres yeux. Si la foi doit s'enraciner dans les réalités spirituelles, transcendantes et invisibles, l'arrivée en Terre Sainte rétablit l'ordre ancien, biblique, des révélations divines dont bénéficie le pèlerin pour entrer en communion immédiate avec Dieu.

Dans le deuxième film, on voit Jürgen Bühler parler depuis Jérusalem. En arrière-plan se profile un grand dessin de Jérusalem biblique où on voit, entre deux oliviers, la tour de David dans la vieille ville. Bühler, en tenue décontractée, réitère la même démarche argumentative qui s'articule autour de la prophétie de Zacharie, citée cette fois-ci dans son intégralité ${ }^{10}$.

Dans le discours de Jérusalem, Bühler s'inscrit dans le genre discursif du sermon en insistant sur une dimension absente du discours d'Abidjan: la promesse de la bénédiction. Lisons ses propos :

La parole de Dieu donne aussi un avertissement mais permettez-moi de la transformer en une bénédiction. Il dit que ces nations qui viennent célébrer la fête des tabernacles, je déverserai ma bénédiction sur eux. Donc, si vous voulez venir de votre nation, de votre ville et de votre communauté, je crois que Dieu veut vous bénir et je crois que Dieu va vous rencontrer ici à Jérusalem d'une manière particulière. [...] Venez à Jérusalem, recherchez le Seigneur et découvrez comment Dieu va bénir votre nation en retour. J'ai hâte de vous voir et de vous accueillir ici dans la Cité de Dieu.

20 Le prédicateur commence par citer le passage de la Bible avant de proposer son explication. Renonçant à l'argumentation par la peur ( « S'il y a des familles de la terre qui ne montent pas à Jérusalem pour se prosterner devant le roi, l'Eternel des armées, 
la pluie ne tombera pas sur elles » Zacharie, chap.14, verset 16-17 »), il tente de séduire son public par la grâce et la faveur divine. Partir en pèlerinage c'est mener une vie plus conforme aux exigences religieuses mais qui permet aussi de bénéficier du trophée symbolique accordé par Dieu en l'image de la bénédiction. Pourtant, celle-ci n'est pas destinée au pèlerin de façon individuelle mais à la «nation » dont il fait partie. L'usage de la notion de "nation" est révélateur de l'image de l'auditoire. Elle se profile à travers une doxa religieuse sollicitée par le prédicateur pour emporter l'adhésion des croyants qui le regardent à la télévision (Amossy 2006). Il convient d'expliquer, dans un premier temps, le sens que peut générer la notion de nation dans le contexte en question, puis de préciser, dans un deuxième temps, l'engagement qu'elle implique visà-vis de l'auditoire.

21 La notion de nation est prolixe en significations qui relèvent à la fois du genre discursif - le discours religieux du sermon chrétien - et de l'univers encyclopédique du destinataire. Si la notion de nation se rapporte souvent, dans le discours social, à des entités sociales et politiques liées à la structuration contemporaine de l'État, elle a aussi des origines discursives profondes dans la foi chrétienne. Elle fait partie d'un champ lexical qui comporte des notions annexes telles que " peuple » et " gentils", traduction des mots hébraïques am et goy (Speiser 1960). Ces mots ne se substituent pas gratuitement l'un à l'autre dans l'Ancient Testament, ils se réfèrent à différentes notions de la société que le texte biblique charge de sens et de conséquences spécifiques (ibid.). Le même procédé discursif opère dans la pensée chrétienne. La notion de nation joue un rôle déterminant pour définir le rôle qu'il faut accorder aux Juifs dans l'histoire sacrée. Selon l'épître de Paul, les «nations » chrétiennes ne sont pas les héritières d'Abraham par le sang, mais elles le deviennent par la foi - c'est la célèbre doctrine chrétienne de la substitution (Longenecker 2007). Le sionisme chrétien s'oppose à une telle théologie de remplacement. La Nouvelle Alliance avec Jésus ne rend pas caduque l'Alliance abrahamique selon laquelle Israël doit devenir une "entité nationale sur sa propre terre ». Le discours religieux s'invite ainsi dans le discours politique : l'État hébreu est perçu comme la réalisation de la promesse biblique de Dieu pour son peuple élu, Israël ${ }^{11}$.

Dans son article «Identités religieuses, appartenance communautaire et participation sociale » (2020), Anne-Sophie Lamine s'efforce d'expliquer ce que veut dire «croire en acte » dans le contexte religieux. Elle évoque trois modalités principales. La première modalité est celle de la "construction de soi ", où le croyant se replie sur lui-même pour vivre une "expérience » individuelle de transmission ou de contemplation, ou pour effectuer un «travail sur soi », qui implique davantage une discipline personnelle - prière, lecture, jeûne... (2020 : 115). La deuxième modalité « renvoie au dépassement de soi ou à l'aspiration à s'élever au-dessus de la satisfaction de ses besoins quotidiens " (ibid.), c'est la dimension transcendante de la pratique religieuse. La troisième modalité est celle "d'être ensemble ou de faire communauté ». Elle se réfère à la dimension sociale inhérente aux rituels de groupes, aux rassemblements religieux et aux relations chaleureuses et solidaires pratiquées entre les membres du groupe. On peut distinguer, avec l'auteur, les deux premières modalités rattachées au monde intérieur du croyant, de la troisième modalité qui se relie à sa vie en société.

23 Les deux discours, celui d'Abidjan puis de Jérusalem, reposent sur les deux aspects du « croire en l'acte »- le monde intérieur et la vie en société - mis en relief dans l'article de Lamine: si Bühler séduit son destinataire, dans son discours de Jérusalem, en 
s'appuyant sur la figure du croyant isolé, soucieux de l'élévation de son âme vers Dieu, son discours d'Abidjan met en relief l'être social du croyant, insistant sur son insertion communautaire. Les deux discours sont ainsi complémentaires : l'expérience spirituelle individuelle du pèlerin, dans le premier discours, est indissociable, dans le deuxième discours, de la collectivité dont il fait partie. Ainsi, une identité prophétique du prédicateur se construit en parallèle de l'identité collective que le prédicateur veut attribuer aux téléspectateurs, membres de cette nation-témoin de l'accomplissement de la prophétie unique que Dieu a réservé à Israël à la fin des temps, c'est-à-dire aujourd'hui ${ }^{12}$. Le prédicateur édifie ainsi une collectivité de croyants pour renforcer la responsabilité impartie à chacun de ses membres. Chaque pèlerin est considéré comme un membre de la "nation" à laquelle il appartient et dont il est redevable. L'identité collective, l'appartenance à la nation, est ainsi mise en valeur pour convaincre le pèlerin de partir en Terre Sainte. Cette image altruiste mise au compte du téléspectateur est censée le faire passer à l'acte de remplir son obligation de chrétien et de futur pèlerin.

Cette première partie s'est intéressée à une interaction télévisée qui s'opère à l'échelle continentale entre un locuteur-prédicateur et son public de téléspectateurs croyant de la chaîne LMTV. Le chapitre suivant nous amène au Bénin. L'interaction analysée s'opère à travers des brochures de publicité que l'agence de voyage Gota distribue à son public chrétien. Cette interaction est conditionnée par le nombre d'affiches distribuées et leur circulation locale et/ou régionale.

\section{Prélude pour un pèlerinage (II) : Gota voyages et le Consulat honoraire d'Israël au Benin}

La date de 2008 est une année charnière non seulement pour les spectateurs africains de LMTV mais aussi pour les chrétiens, partisans d'Israël, au Bénin. En cette année, M. Raïmi Issa Lawani, ancien colonel de l'armée béninoise, fonde le Consulat honoraire d'Israël au Benin et devient par là-même son premier Consul. Interviewé dans le cadre de cette recherche, M. Franck Agbede, Chargé de Mission auprès du Consul, indique que l'attachement que le premier consul témoigne pour Israël s'enracine dans son parcours professionnel de soldat formé en Israël pendant six mois durant la période ultérieure à la Guerre des six jours. D'après $M$. Agbede, les pèlerinages en Terre Sainte ne commencent qu'en 2012, avec la nomination du Vice-Consul, Mme Grâce Gangbo Lawani, l'épouse du Consul, au moment où s'achève sa nomination ministérielle de Chargé de Mission auprès du Président de la République.

Les voyages de pèlerins sont organisés avec le concours de l'agence de voyage Gota, située à Cotonou. Le Consulat s'occupe des problèmes consulaires et peut intervenir auprès de l'ambassade d'Israël pour l'étude des dossiers en cas de demande de visa individuel. L'envoi des pèlerins en Terre Sainte s'inscrit dans un cadre plus large d'activités religieuses promues par le Consulat sous l'inspiration du sionisme chrétien. Parmi ses nombreuses activités, le Consulat prend en charge la construction des églises, vient en aide aux couches défavorisées et à leurs congrégations, organise chaque année la fête du Tabernacle en concordance avec les dates hébraïques; car les chrétiens, explique M. Agbede, « ont compris que celui qui s'attache à Israël, eh bien s'attache à son Dieu. Partout dans le monde, on n'a jamais appris Dieu d'un autre pays si ce n'est celui d'Israël : Dieu d'Israël ». 


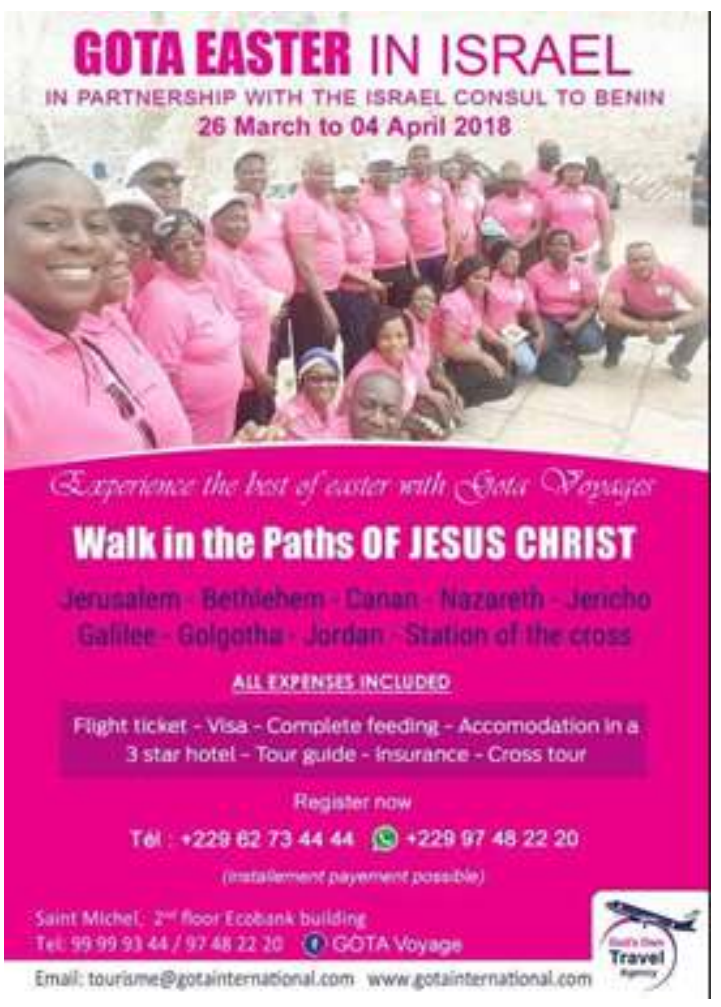

Affiche des voyages de pèlerins de l'agence de voyage Gota 2018

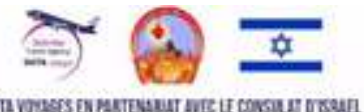

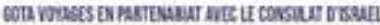

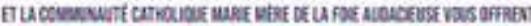

GOTA PÂQUES EN ISRAEL

(JERUSALEM POUR TOUS)

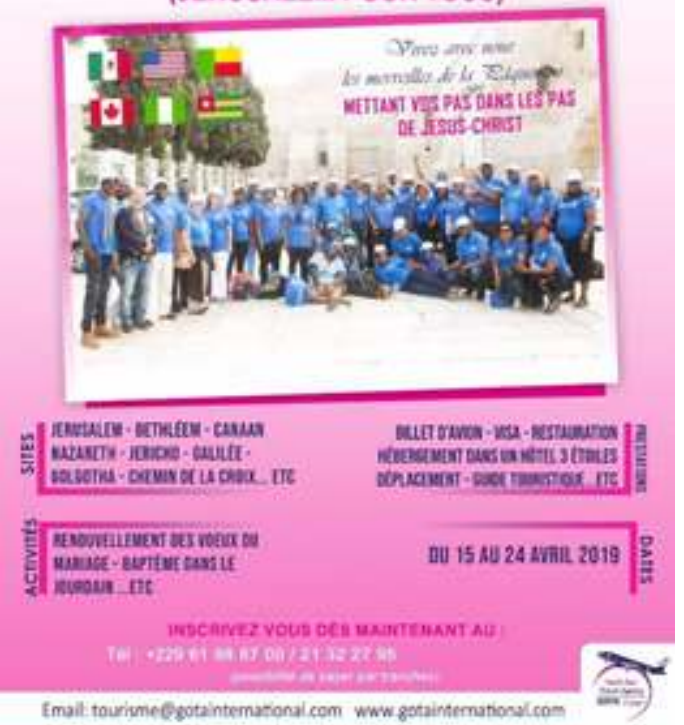


Affiche des voyages de pèlerins de l'agence de voyage Gota 2019

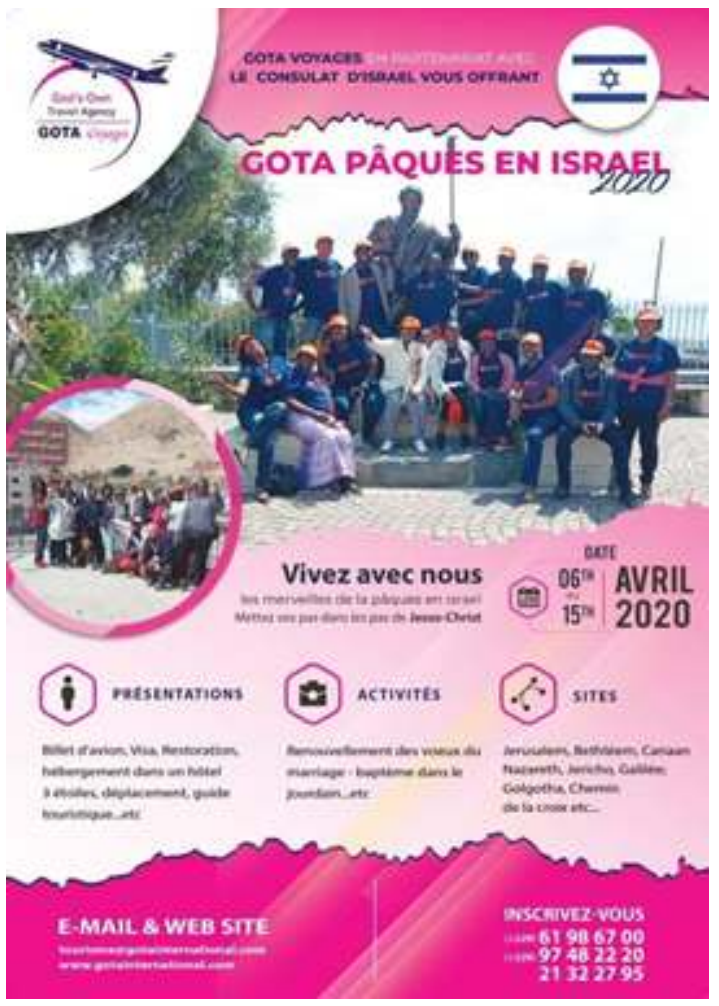

Affiche des voyages de pèlerins de l'agence de voyage Gota 2020

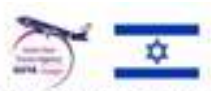

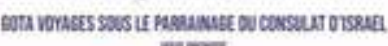
minnom

\section{GOTA PAQUES EN ISRAEL}

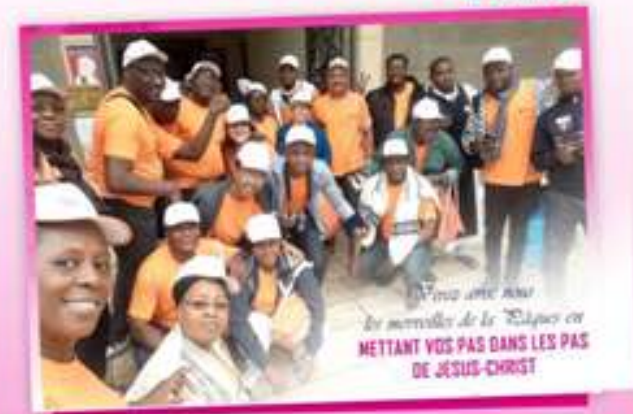

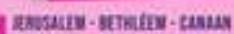

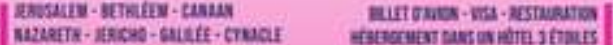

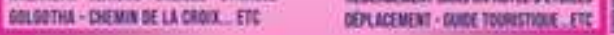

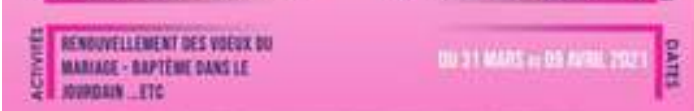

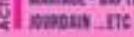

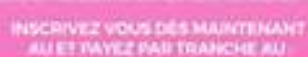

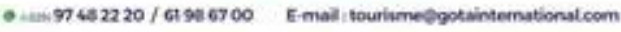



marché du tourisme religieux. L'organisatrice, l'agence de voyage Gota, respecte bien ces consignes. Elle diffuse les affiches publicitaires pour promouvoir l'information et encourager les croyants à participer aux voyages de pèlerins en Terre Sainte. Le discours publicitaire s'apparente au genre épidictique (Adam et Bonhomme 1997 : 19) on peut le ranger dans les discours à visée persuasive, comme le prêche à l'église ou le discours de campagne électorale (Amossy 2006: 34). L'étude de ces publicités se propose de montrer comment les institutions religieuses béninoises se livrent au jeu de l'industrie du tourisme et de la publicité, afin de construire une image conforme à une logique d'achat et de consommation visant une très vieille pratique religieuse à haute valeur symbolique telle que le pèlerinage en Terre Sainte.

constate, d'entrée de jeu, la mise en page commune aux quatre affiches. Dans toutes les quatre, les informations encadrent une image photographiée - un portrait du groupe - posée au centre de l'affiche. Roland Barthes, un pionnier de la sémiologie de l'image publicitaire, étudie, dans son article-phare de 1964 La Rhétorique de l'image, les messages possibles que l'image publicitaire est conviée à véhiculer. Il indique " qu'en publicité, la signification de l'image est assurément intentionnelle» (1964:40). Les affiches publicitaires qui nous intéressent ne font pas exception à la règle : pour vendre le voyage de pèlerins, elles essaient de faire adhérer les croyants à une certaine perception de l'expérience qu'ils vont vivre en tant que pèlerins en devenir.

Dans le sillage de Barthes, Adam et Bonhomme s'intéressent aux rapports établis entre le texte et l'image pour donner une définition opératoire au dispositif publicitaire. Il s'agit d'une

structure sémiologique mixte en raison des latitudes de dosage qu'il permet entre le texte et l'image et surtout de la diversité de ses composantes. Ces constituants fournissent par ailleurs autant de bases signifiantes sur lesquelles se greffent les [...] facteurs de l'efficacité argumentative du genre publicitaire (Adam et Bonhomme $1997: 55)$.

Le choix de l'image est donc un facteur important de «l'efficacité argumentative » de l'affiche et de la réussite du message publicitaire. Pour dégager la valeur argumentative de l'image, il faut prendre en ligne de compte que le processus cognitif de l'interprétation est «fortement culturel» (Barthes). Les portraits de groupe représentés sur les affiches publicitaires véhiculent une certaine vision du monde et doivent être interrogés comme une construction sociale singulière (Cohen et Ramel 2016). L'image est donc un message susceptible de donner des raisons pour ou contre un point de vue (Blair $1996 ; 2004)$.

31 Mais ces images pourraient être banales, figurer ailleurs sur n'importe quelle publicité pour exalter une expérience touristique de groupe, vers quelque destination que ce soit. La question qui se pose d'emblée se réfère au choix des portraits de groupe pour séduire le futur pèlerin à effectuer les voyages proposés. Pourquoi présenter un groupe et non pas, par exemple, un des sites célèbres de Jérusalem dont rêvent les fidèles quand ils lisent la Bible? Pourquoi choisir une collectivité de pèlerins au détriment du fidèle isolé accomplissant son but spirituel, à savoir s'approcher de Dieu, communiquer avec Lui?

32 Pour donner quelques éléments de réponse, il convient de s'interroger sur le sens que génère la juxtaposition du texte et de l'image, avant de procéder à l'analyse du

Argumentation et Analyse du Discours, 27 | 2021 
« portrait de groupe » par le genre qui détermine sa « nature communicationnelle » et ses « régularités compositionnelles » (Charaudeau 2002).

\subsection{Rapport texte-image}

Dans l'affiche publicitaire, le texte et l'image jouent donc un rôle complémentaire. La démarche persuasive résulte de l'effet produit par les liens sémiotiques qui se nouent entre eux (Iváñez 2018). On s'intéressera d'abord au contenu textuel des affiches.

A partir de 2019, les informations sont réparties en quatre catégories - date, prestation, sites, activités. Cette catégorisation donne du produit l'image d'un package touristique complet. Elle assure, d'une part, les aspects procéduraux et les conditions adéquates du voyage (date et prestation), et garantit d'autre part une expérience religieuse digne de ce nom. La catégorie "activité » a une fonction évocatrice. "Baptême dans le Jourdain » s'adresse à la dévotion du croyant isolé en l'incitant à se voir dans l'eau sacrée du Jourdain. Mais cette catégorie élargit le public-cible au-delà du croyant isolé. Elle s'adresse aussi au couple. Le "renouvellement des vœux du mariage " est une pratique très récente, qui commence dans les années 1990 aux États-Unis (Braithwaite et Baxter 1995), et s'est étendue depuis dans le monde entier. L'évocation du renouvellement des vœux de mariage en Terre Sainte est ici une astuce publicitaire dont la force persuasive réside dans l'imbrication de la culture populaire dans l'univers religieux. L'expérience religieuse s'approprie une dimension romantique dont peuvent bénéficier les couples mariés.

La catégorie «sites » comprend des noms de lieux - «Jérusalem, Bethléem, Canaan, Nazareth, Jéricho, Galilée, Golgotha, Chemin de la Croix... etc. » - qui jouent un double rôle métonymique de l'histoire biblique et des conditions particulières (cultes religieux) de sa lecture. La figure de style de l'accumulation crée un effet d'amplification, censée frapper le consommateur par une impression d'exhaustivité selon le topos bien connu du meilleur rapport qualité/prix.

Comme le design caractéristique de chaque affiche, la composition textuelle varie aussi d'une année à l'autre. Certaines informations restent constantes, d'autres changent. Le titre, dans toutes les affiches, « Gota Pâque en Israël » transforme le voyage de pèlerins en produit commercialisé. La particularité de la fête ne résulte plus seulement de son aspect religieux, universalisé, mais aussi de sa dimension particulière, reliée à la marque Gota à laquelle la fête est associée. L'invitation que l'agence adresse au public dans l'affiche de 2021 - «Vivez avec nous les merveilles de la Pâque en mettant vos pas dans les pas de Jésus-Christ»-implique un rapport de causalité : les merveilles de la Pâque ne peuvent se révéler qu'avec une ascèse de communion avec le Christ qui s'opère dans l'acte de marcher dans ses pas.

37 Le texte et la photo sont complémentaires, leur juxtaposition renforce le message publicitaire. Le texte renvoie le sens dénotatif de l'image - hommes et femmes rangés sur deux lignes, debout et assis, portant une chemise de la même couleur - à son sens connotatif - ceux-ci sont des pèlerins voyageant en Terre Sainte. Le «nous » du texte ne se réfère plus au «nous » de l'agence organisatrice mais aux pèlerins qui appellent leurs frères et sœurs dans la Foi à suivre leur vocation religieuse (« marcher sur les pas de Jésus dans la Terre Sainte »). Inversement, les pèlerins dans la photo deviennent garants de la véracité du texte et donc de la qualité du « produit » promu. Il s'agit ici 
d'une rhétorique de l'image par l'exemple: si «nous» sommes satisfaits de notre voyage en Terre Sainte, vous allez l'être aussi.

\subsection{Le portrait de groupe et la question du genre}

Dans son article sur le portrait de groupe dessiné dans la Hollande du $18^{\mathrm{e}}$ et du $19^{\mathrm{e}}$ siècle (2004), Aloïs Riegl montre comment le portrait de groupe évolue dans l'histoire de l'art plastique en une représentation de plusieurs membres autonomes dans une "corporation volontaire» (2004: 39). Cette «corporation volontaire" crée une apparence d'unité ou de cohésion groupale au détriment de l'autonomie individuelle, sans pourtant l'effacer complètement (ibid.).

39 A l'ère digitale des réseaux sociaux, la prise des photos (portraits) de groupe est devenue un rituel courant dont tout le monde connait bien le scénario. Chacun doit s'investir pour la réussite de la photo. Regard souriant et gestuelle sont nécessaires pour donner l'impression que l'expérience vécue par les membres du groupe est d'autant plus significative qu'elle mérite d'être prise en photo. Si la photo capte le moment, elle valide aussi sa singularité qui, en retour, lui accorde sa légitimité. Autrement dit, si le moment appelle la photo, cette dernière institue le moment, l'établit officiellement et durablement. Un accord tacite s'installe entre les membres du groupe pris dans le jeu théâtral devant la caméra. La prise de photo est ainsi un acte ritualisé et institutionalisé, elle valide les liens que les personnages figurés ont noués en cours de route. La photo est une sorte de confirmation de la transformation des individus isolés en groupe soudé.

Si l'on revient à une analyse plus détaillée du portait de groupe, on constate des caractères communs à la mise en scène des quatre portraits. Chaque photo se réfère à une promotion qui se distingue par une couleur emblématique, en contraste avec l'arrière-plan. Celui-ci donne à la photo une sensation de dépaysement grâce aux cadres historiques (arches, 2018) et aux cyprès emblématiques de la Méditerranée (2019) qui se profilent derrière eux. Aucune indication n'est mentionnée au sujet des sites visités. Les croyants africains doivent faire confiance au fait que ces photos ont bien été prises en Terre Sainte ${ }^{13}$. Le décor de l'image dans l'affiche publicitaire de 2021 - entrée de bâtiment - relève d'un choix complètement arbitraire, profane. Dans l'affiche de 2020, les pèlerins entourent une statue (St. Pierre) en l'intégrant au groupe, donnant à la photo une dimension humoristique. On voit bien comment l'arrière-plan de ces quatre affiches s'efface pour mettre en valeur le portrait de groupe.

Les portraits présentés sur les affiches de 2018 et de 2021 sont des selfies. Le choix de publier un selfie active une série d'idées reçues. Les selfies sont généralement pris sur le vif, ils sont de nature décontractée ou faits pour paraître décontractés ${ }^{14}$. La disposition selfie donne ainsi à la photo une touche de spontanéité et d'authenticité. Pourtant, quand on juxtapose ces photos en série, on a l'impression que l'agence Gota dispose d'un album de portraits des groupes qu'elle a amenés en Terre Sainte. Plus précisément, la mise en scène commune aux quatre portraits et la disposition des pèlerins en groupe laisse penser qu'il n'y a rien de spontané dans ces portraits; prendre une photo du groupe est un moment ritualisé au sein du voyage de pèlerins organisé par l'agence Gota, et participe d'une stratégie de marketing menée par l'agence de voyage dont l'analyse sémiotique et argumentative de l'image permet de dessiner les contours. 
42 En effet, ce qui se joue dans les portraits n'est ni la dévotion du groupe, ni la piété individuelle. La photo se donne à voir en tant que souvenir de l'ambiance conviviale ou des amitiés que les pèlerins ont réussi à nouer durant le voyage. La mise en scène du portrait de groupe est ainsi constitutive d'une construction sociale appelée à représenter moins l'expérience spirituelle que le groupe soudé qui l'a rendue possible.

On voit l'enjeu : bien que le langage verbal soit considéré comme la norme pour penser l'argumentation en général, il est tout à fait possible de penser l'existence d'une orientation argumentative dans les images qu'on vient d'analyser (Roque 2019). La dimension sociale de ces portraits de groupe participe à une valeur constitutive de l'ethos collectif (Orkibi 2008) des pèlerins, construit au gré d'un réel besoin persuasif: influencer les croyants africains à partir en voyage de pèlerins avec l'agence Gota. Cet ethos collectif insiste moins sur la vocation du pèlerin à marcher sur les pas de Jésus en Terre Sainte et à y accomplir des rituels religieux (prières, baptême etc.), que sur des valeurs sociales de fraternité, d'amitié, et de vie en communauté, conditions de la réussite de son expérience religieuse.

\section{Pèlerins en route sur la Terre Sainte : le support des objets matériels}

Le chapitre qui suit s'intéresse à la construction discursive de l'expérience du pèlerinage en Terre Sainte. Cette expérience résulte de la présence physique du pèlerin dans les lieux de culte, et se transforme immédiatement en discours produit sur cette présence. En d'autres termes, l'expérience du pèlerinage est un discours narrativisé que les pèlerins produisent durant et après leurs visites dans les lieux de culte. Mais ce discours est nourri aussi par des biens matériels - souvenirs, photos, artefacts - que le pèlerin accumule en cours de route. Ces matériaux ont une valeur performative et participent de la construction discursive de l'expérience vécue par le pèlerin. Donnant de la substance à la mise en discours du voyage de pèlerins, l'objet matériel offre des repères sur l'itinéraire parcouru, permet à chacun de s'approprier l'expérience vécue et s'insère dans l'habitus religieux et culturel du pèlerin de retour dans son pays d'origine (Thurlow et Jaworski $2010: 8$ ). 


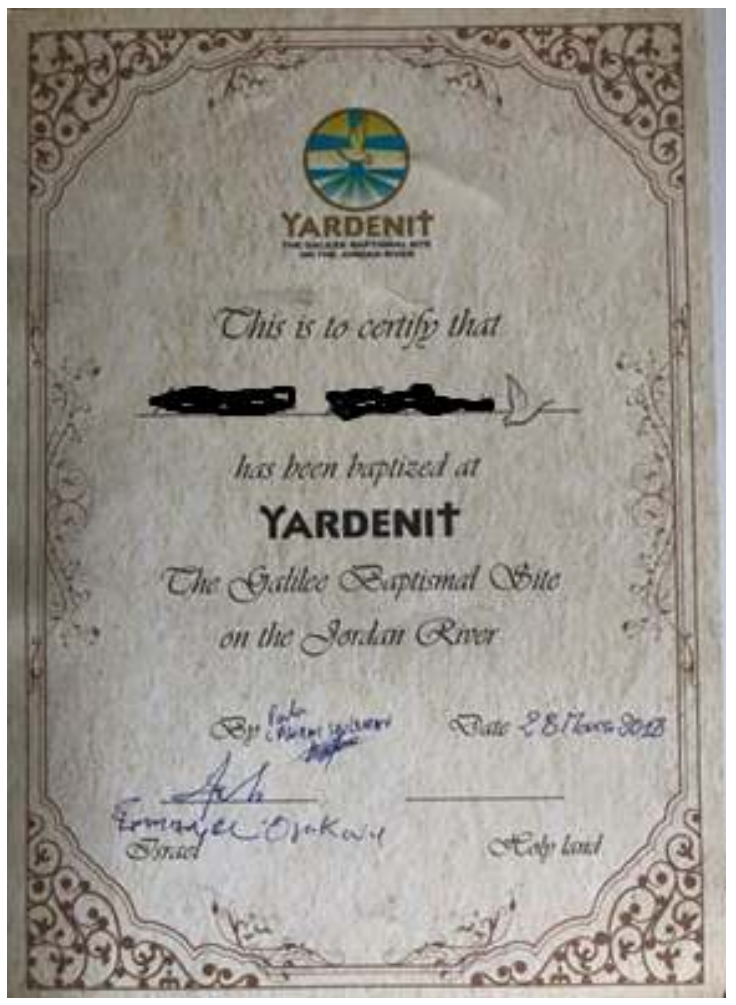

Une attestation de passage sur le site baptismal de Yardenit

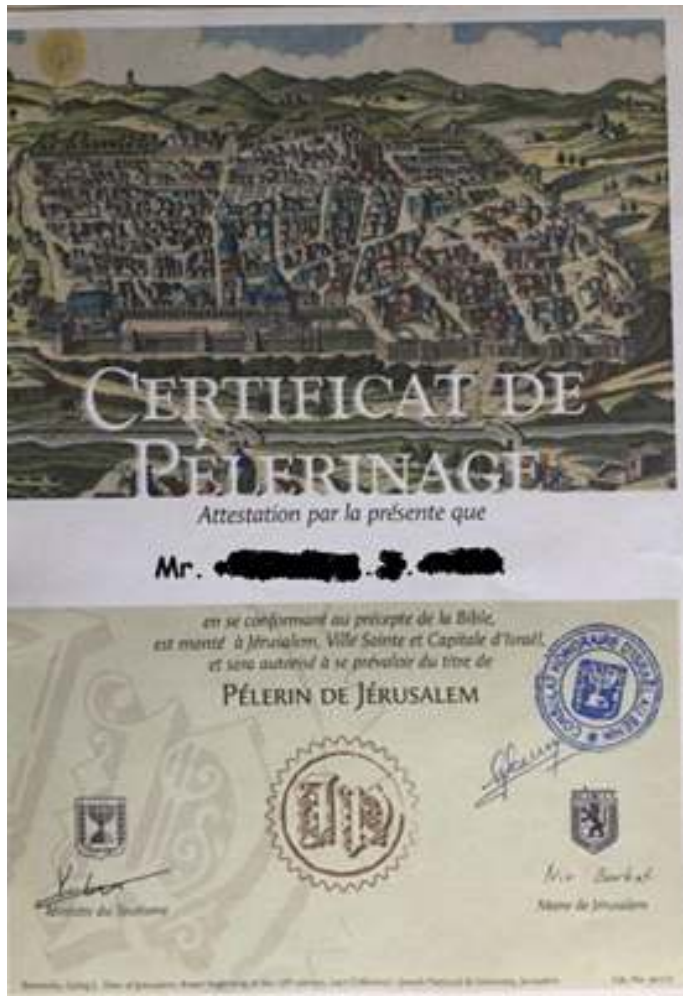

\section{Certificat de Pèlerinage}

Ces deux attestations n'ont pas la même valeur symbolique. Une attestation rédigée en anglais vise un large public, elle est accordée à chaque pèlerin de passage sur le site 
baptismal de Yardenit. Le certificat aide à valider l'authenticité de ce site imaginé par le ministère du tourisme dans les années 1970 (Ron et Feldman 2009). Si la première attestation confirme la performance du pèlerin in situ (le baptême dans le Jourdain), le " certificat de Pèlerinage » se réfère à l'intégralité de son voyage. L'agence israélienne de voyage Talya tours, qui collabore avec Gota pour assurer le pèlerinage en Israël, décerne le certificat lors d'une cérémonie festive à l'hôtel, la veille du départ. Cette scénographie d'une cérémonie de clôture singularise le certificat que l'État d'Israël décerne, par l'intermédiaire de l'agence de voyage, à chacun des pèlerins en l'honorant pour l'expérience qu'il vient d'accomplir ${ }^{15}$. Sa légitimité découle de la signature de M. Nir Barkat, maire de Jérusalem, et de celle de M. Yariv Levine, ministre du Tourisme, et des symboles iconiques de la ville de Jérusalem - le lion, et celui de l'État d'Israël - la ménora. Ces signatures sont d'autant plus importantes qu'elles proviennent de hautes autorités officielles de l'État. Cette implication de l'État hébreu dans le projet religieux du pèlerinage s'inscrit en filigrane dans le discours du christianisme sioniste, qui relie la prophétie biblique au projet national de la construction de l'État d'Israël. Ce document de l'État hébreu, validé par les signatures de ses représentants, prend ainsi un sens religieux, chrétien, qui s'accorde avec le texte écrit pour accroître sa légitimité :

Attestation par la présente que Mr. en se conformant au précepte de la Bible, est monté à Jérusalem, Ville Sainte et Capitale d'Israël, et sera autorisé à se prévaloir du titre de Pèlerin de Jérusalem.

Reproduisant le cadre doctrinal du christianisme sioniste, le texte entremêle un registre de discours géopolitique - Jérusalem «capitale d'Israël» - et un discours religieux et traditionnel de la culture judéo-chrétienne - Jérusalem «Ville Sainte ». Les symboles iconiques et les signatures redoublent ainsi la référence implicite dans le texte, porteur de l'idéologie politique du christianisme sioniste qui sous-tend la création et l'épanouissement de l'État hébreu. Ce dispositif idéologique sous-jacent valide ipso facto l'adhésion du pèlerin à la mouvance évangéliste du sionisme chrétien. Cet attachement idéologique est d'autant plus significatif que M. Franck Agbede précise la valeur symbolique de l'attestation qu'il "garde précieusement", comme le font, d'après lui, les autres pèlerins.

Le cachet du "Consulat honoraire d'Israël au Bénin » accorde un sens supplémentaire à cette attestation, il renforce le pacte entre Israël et le Bénin ainsi qu'entre le pèlerin et le cadre institutionnel qui forge son expérience. L'image biblique de Jérusalem, empruntée au tableau de Georg J. Borowsky, date du début du $18^{\mathrm{e}}$ siècle. Elle inscrit le voyage de pèlerins dans l'histoire biblique, et accorde à l'attestation son apparence authentique. Ce certificat qui atteste de la présence avérée du pèlerin dans la ville Sainte de Jérusalem, implique des privilèges symboliques, selon une logique méritocratique. Les lettres JP forment un sigle : «Jérusalem Pèlerin. » Ce sigle devient une marque distinctive pour les pèlerins partis avec l'agence Gota et le Consulat d'Israël au Bénin. 


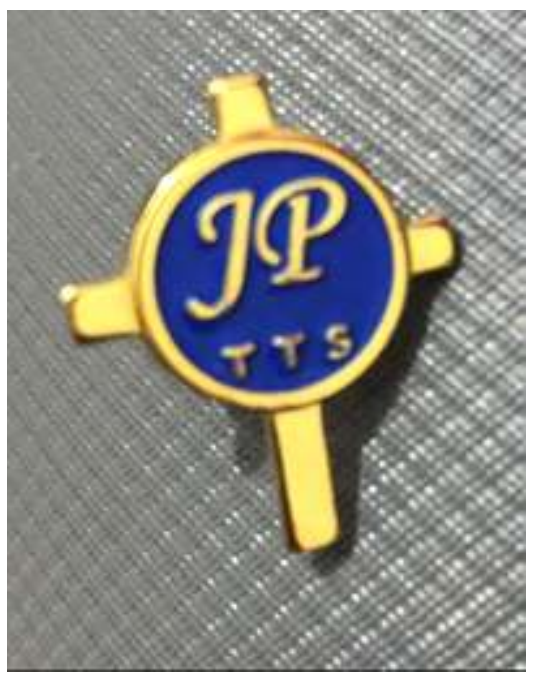

Pin décerné avec l'attestation « Pèlerin de Jérusalem » par l'agence de voyage Talia tours (TTS) voyage Talia tours (représenté par le sigle TTS) la veille du départ pour les pays d'origine. D'après M. Franck Agbede, les pèlerins portent cette décoration honorifique, placée sur l'habit au niveau de la poitrine, non loin du cœur, lors d'une cérémonie officielle organisée par le Consulat et l'agence Gota après le retour au Bénin. Cette cérémonie festive et médiatisée, en présence de la famille et des amis des pèlerins, inclut des «témoignages très émouvants, [la présentation] des photos d'ensemble, des images et vidéos des sites et des lieux visités en Israël depuis l'arrivée jusqu'au départ. "

Cette décoration et l'honneur qu'elle confère reproduit le scénario des clubs d'élites qui se forgent des symboles pour se reconnaitre et se distinguer des autres. En effet, chaque voyage de pèlerins supplémentaire accorde au pèlerin le droit à un nouveau pin. Ainsi, les nouveaux pèlerins de retour au pays ont droit à porter un pin et rejoignent les membres du groupe qui le portent déjà. Une échelle hiérarchique s'établit entre les membres du groupe selon le nombre de voyages déjà exécutés. $\mathrm{M}$. Agbede insiste sur l'importance symbolique accordée au pin, une source de fierté, dit-il, de

montrer à tous qu'on a déjà été en Israël. Puisque rien d'autre ne pourra montrer à celui qui vous rencontre que vous avez foulé le sol israélien une fois. Mais avec le pin sur votre poitrine au cours des grandes cérémonies ou des rencontres nationales, on le découvre en comptant le nombre de pins sur votre poitrine (comme certains qui ont 5 pins sur leur col).

Le pin et les attestations marquent ainsi l'incorporation de l'individu dans ce groupe social, religieux. La cérémonie est un acte performatif qui donne son cachet à l'initiation du croyant dans le groupe sélectif des pèlerins. Si les pèlerins jouissent d'un statut important dans leur communauté respective, celui-ci est accompagné de privilèges, conformément aux règles génériques de cette pratique sociologique de rite d'initiation. A M. Agbede de conclure :

Après le pèlerinage, la plupart des participants reçoivent un fait nouveau (sic) à leur avantage, ou une bonne nouvelle, ou une quelconque satisfaction ou encore une promotion, une promotion de grade professionnel ou religieux, ou un dénouement de situation ou d'affaire etc. Sommes toutes, chacun reçoit toujours une portion 
nouvelle de joie. Voilà ainsi autant d'avantages dont peuvent bénéficier les pèlerins de retour au pays. C'est un fait très courant et autant de fois va-t-on à Jérusalem, autant de bonnes nouvelles dans sa vie ou celle de ses proches.

\section{Conclusion}

51 Au terme de cette analyse, on peut constater jusqu'à quel point l'expérience offerte aux pèlerins utilise la coopération de forces sociales diverses. Le monde ecclésiastique travaille avec le milieu diplomatique, l'industrie du tourisme, le monde de la publicité et des communications de masse. Cette action collective, synergique, émerge à partir de la montée en puissance de l'Église évangélique et pentecôtiste en Afrique.

En effet, si on peut qualifier l'Afrique de "laboratoire créateur » (Jenkins 2011) de nouvelles formes de christianisme, les mouvances évangéliques et pentecôtistes jouent un rôle important dans cette perpétuelle reconfiguration religieuse et culturelle (Fath et Mayrargue 2014, Adogame 2013). On peut voir, au terme de cette recherche, comment l'entreprenariat prosélyte qui se constitue autour des pèlerinages propose un nouveau capital social aux chrétiens dans la région de l'Afrique occidentale francophone. Si on relie souvent le chemin de pèlerinage à la piété individuelle des pèlerins, notre analyse dévoile la capacité du voyage à générer une image de communauté à base de différentes valeurs sociales, idéologiques et religieuses. Une image de communauté se forge, dans le discours évangélique de Jürgen Bühler, qui s'organise autour de l'idée de la nation. Elle se présente ensuite à travers la formation d'une communauté de pèlerins basée sur des valeurs d'amitié fraternelle, qui conditionnent la réussite de l'expérience spirituelle. Enfin on insiste sur la capacité du voyage de pèlerins à former une couche d'élite sociale, qui se crée dans un espace de sociabilité sélective à partir de rites initiatiques. Cette nouvelle "élite voyageuse ", soudée autour d'un nouvel idéal national calqué sur la doctrine évangélique, aurait-elle en vue d'être, à plus long terme, un facteur d'influence majeur sur les sociétés locales en évolution instable dans les démocraties émergentes d'Afrique sub-saharienne?

\section{BIBLIOGRAPHY}

Adam, Jean-Michel \& Marc Bonhomme. 2011. L'argumentation publicitaire: Rhétorique de l'éloge et de la persuasion (Paris : A. Colin)

Amossy, Ruth. 2006. L'argumentation dans le discours (Paris : A. Colin)

Adogame, Afe. 2013. The African Christian Diaspora. New Currents and Emerging Trends in World Christianity (London : Bloomsbury)

Barthes, Roland. 1964. « Rhétorique de l'image », Communication 4, 41-42

Blair, Anthony J. 1996. "The Possibility and Actuality of Visual Arguments", Argumentation and Advocacy 33, 23-39

Argumentation et Analyse du Discours, 27 | 2021 
Blair, Anthony J. 2004. “The Rhetoric of Visual Arguments”, Defining Visual Rhetoric, eds. Charles A. Hill \& Marguerite Helmers. (Mahwah, New Jersey, London: Laurence Erlbaum Associates), $41-62$

Braithwaite, Dawn O. \& Leslie A. Baxter. 1995. « "I Do” Again: The Relational Dialectics of Renewing Marriage Vows », Journal of Social and Personal Relationships 12, 177-198

Charaudeau, Patrick. 2002. « Genre », Dictionnaire d'analyse du discours (Paris : Seuil), 277-280

Cohen, Corentin \& Frédéric Ramel. 2016. « Prendre les images au sérieux Comment les analyser?», Devin, Guillaume (éd). Méthodes de recherche en relations internationales (Paris : Presses de Science Po), 71-92

Dorier-Apprill, Élisabeth \& Robert Ziavoula. 2005. « La diffusion de la culture évangélique en Afrique centrale », Hérodote 119, 129-156

Feldman, Jackie. 2017. « Key figure of mobility: the pilgrim », Social Anthropology 25, 69-82

Flood, David. 1983. Frère François et le mouvement franciscain (Paris : Editions Ouvrières)

Engberg, Aron. 2020. «Evangelical Zionism in Jerusalem », Walking on the Pages of the Word of God: Self, Land, and Text Among Evangelical Volunteers in Jerusalem (Leiden : Brill), 39-74

Fath, Sébastien. 2005. « Le poids géopolitique des évangéliques américains : le cas d’Israël », Hérodote 119, 25-40

Fath, Sébastien \& Cédric Mayrargue. 2014. « Les nouveaux christianismes en Afrique. Introduction thématique ", Afrique contemporaine 252, 13-26

Iváñez, Montserrat P. 2018. « Le poids des mots et des images dans les textes de promotion touristique », Konowska, Agnieszka, Agnieszka Woch, Andrzej Napieralski \& Anna Bobińska (éds). Le poids des mots. Hommage a Alicja Kacprzak (Łódź : Presses universitaires de Łódź), 229-238

Jaworski, Adam \& Crispin Thurlow. 2010. Tourism Discourse: Language and Global Mobility (Basingstoke \& New York : Palgrave MacMillan)

Jenkins, Philip. 2011. The Next Christendom: The Coming of Global Christianity (Oxford : Oxford U. P.)

Lamine, Anne-Sophie. 2020. «Identités religieuses, appartenance communautaire et participation sociale », Revue du droit des religions 10, 105-121

Longenecker, Bruce. 2007. « On Israel's God and God's Israel: Assessing Supersessionism in Paul », The Journal of Theological Studies 58, 26-44

Maingueneau, Dominique. 2009. «Le sermon : contraintes génériques et positionnement », Langage et société $130,37-59$

Mayrargue, Cédric. 2014. «Les christianismes contemporains au Bénin au défi de la pluralisation. Dynamiques d'expansion et porosité religieuse », Afrique contemporaine 252, 91-108

Merkley, Paul C. 1998. The Politics of Christian Zionism 1891-1948 (London : Franck Cass)

Miran-Guyon, Marie. 2014. « Apocalypse patriotique en Côte d'Ivoire. Le pentecôtisme de la démesure ", Afrique contemporaine 252, 73-90

Morinis, Alan. 1992. Sacred Journeys: the Anthropology of Pilgrimage (Westport, Conn. : Greenwood Press)

Orkibi, Eithan. 2008. «Ethos collectif et Rhétorique de polarisation : le discours des étudiants en France pendant la guerre d'Algérie ", Argumentation et Analyse du Discours 1, https://doi.org/ $10.4000 /$ aad.438 
Rabatel, Alain. 1998. La construction textuelle du point de vue (Neuchâtel : Delachaux et Niestlé)

Raynal, Sandrine. 2005. « En Côte d'Ivoire, 'les forces du bien contre les forces du mal' », Hérodote $119,111-128$

Riegl, Eloïs. 2004. « Une bonne image de soi. Portraits de groupe et ethos calviniste », Actes de la recherche en sciences sociales $154,36-45$

Rinschede, Gisbert. 1992. « Forms of Religious Tourism », Annals of Tourism Research 19, 51-67

Ron, Amos S. \& Dallen J. Timothy. 2019. Contemporary Christian Travel: Pilgrimage, Practice and Place (Bristol : Channel View Publications)

Ron, Amos S. \& Jackie Feldman. 2009. « From spots to themed sites - the evolution of the Protestant Holy Land », Journal of Heritage Tourism 4, 201-216

Roque, Georges. 2019. « Comment argumenter à partir d'images ? », Signata. Annales des sémiotiques 10, https://doi.org/10.4000/signata.2363

Speiser, Ephraim A. 1960. «'People' and 'Nation' of Israel », Journal of Biblical Literature 79, 157163

Stockton, Ronald R. 1987. « Christian Zionism: Prophecy and Public Opinion », Middle East Journal $41,234-253$

Tomasi, Luigi. 2002. « Homo Viator: From Pilgrimage to Religious Tourism via the Journey », Swatos, William H. Jr. and Luigi Tomasi (éds). From Medieval Pilgrimage to Religious Tourism (Westport : Praeger Publishers), 1-24

Turner, Victor. 1973. «The center out there: the pilgrim's goal », History of Religions 12, 191-230

Turner, Victor \& Edith Turner. 1978. Images and Pilgrimage in Christian Culture: Anthropological Perspectives (New York : Columbia U. P.)

\section{NOTES}

1. Je voudrais exprimer toute ma gratitude à MM. Franck Agbede et Dieudonné Gollet pour leur coopération assidue, constructive et conviviale sans laquelle cette recherche n'aurait pu se réaliser. Je voudrais aussi remercier l'équipe de l'Ambassade israélienne en Côte d'Ivoire, et tout particulièrement l'ambassadeur en exercice Leo Vinovezky et l'ancien ambassadeur le Dr. Eli Ben Tura pour les informations qu'ils ont bien voulu me fournir. Je remercie aussi M. Yoni Shapira pour m'avoir mis en contact avec les acteurs du tourisme africain et Prof. Amos Ron pour son soutien scientifique tout au long de cette recherche. Last but non least, je voudrais remercier le Prof. Galia Yanoshevsky pour sa confiance et son inlassable soutien.

2. https://www.sciencespo.fr/enjeumondial/fr/odr/afrique-subsaharienne-et-sionismeevangelique.html (consulté le 13 Juillet 2021)

3. Document en ligne : https://www.sciencespo.fr/enjeumondial/fr/odr/afrique-subsaharienneet-sionisme-evangelique

4. Il montre comment ces caractères peuvent se repérer dans la musique gospel, dans l'usage des termes hébraïques pour nommer les gens ou les Églises. L'iconographie juive - la ménora, l'étoile de David ou le drapeau d'Israël - sont aussi très visible dans les espaces culturels évangéliques.

5. D'après son directeur, la chaîne compte autour de huit millions de téléspectateurs à l'échelle continentale.

6. Cette volonté se réalise - au moins partiellement, faute de contenu - grâce au soutien de l'Ambassade d'Israël en Côte d'Ivoire, avec qui la chaîne a pu nouer de très fortes relations, ainsi 
qu'avec la chaîne francophone i24, soucieuse de la circulation d'une image positive de l'État hébreu dans les pays Africains.

7. V. la page du site https://sareltours.com/article/tour-operator-israel (consulté le 04 avril 2021)

8. Sur l'activité de l'ICEJ, voir son site très élaboré (https://int.icej.org/ consulté le 28 juillet 2021) ainsi que l'article récent de Aron Engberg sur le sionisme évangélique à Jérusalem (2020).

9. Susan F. Harding parle d'une « rhétorique de la conversion » pour relier le genre du sermon à sa visée argumentative. D'après elle, la conversion est un procédé rhétorique porté sur la transformation de soi que doivent subir les âmes perdues, à partir d'une méthode censée provoquer ce changement chez ceux qui l'écoutent. En ce sens, le sermon ne serait point un monologue qui constitue son locuteur en tant que personne culturellement spécifique, mais plutôt un dialogue qui reconstitue ses auditeurs (Harding 1987 : 167).

10. "Tous ceux qui resteront de toutes les nations venues contre Jérusalem monteront chaque année pour se prosterner devant le roi, l'Éternel des armées, et pour célébrer la fête des tabernacles. S'il y a des familles de la terre qui ne montent pas à Jérusalem pour se prosterner devant le roi, l'Éternel des armées, la pluie ne tombera pas sur elles.» (Zacharie, chapitre 14 verset 16 à 17)

11. Voir la page «la théologie de la substitution " consacrée à cette question sur le site de l'Ambassade: https://fr.icej.org/la-th\%C3\%A9ologie-de-la-substitution (consulté le 07 juillet 2021).

12. Cette prophétie est mise en scène dans la bande-annonce par la marche des Nations aux côtés d'Israël, représentées, chacune, par son drapeau respectif.

13. Pour situer les sites, je me suis adressé à M. Yoni Shapira, Président de Moreshet Derech, Association des guides touristiques d'Israël, que je remercie chaleureusement. Yoni a pu reconnaitre aisément le site de Capharnaüm (2020), il était moins catégorique au départ pour l'image de 2019 (Bethlehem) et n'a pas reconnu le site dans la photo de 2020. 2018, 2019 Bethlehem (cours de l'église de la Nativité). 2020 - Caphernaum. 2021 - Mont de Beatitudes.

14. Ces idées reçues sont répertoriées sur le site de Wikipedia - une base de données dont l'influence sur la production et la circulation du savoir dans le discours social ne saurait être surestimée.

15. Il faut préciser que ces certificats sont produits en masse par le ministère du Tourisme et disponibles pour toute entreprise qui souhaite les acheter pour leurs pèlerins sur le site du ministère (Ron and Feldman 2009).

\section{ABSTRACTS}

This paper examines a cultural phenomenon that has drawn little attention in the research on religious tourism in the Holy Land: the pilgrimages from francophone sub-Saharan Africa. This study of the pilgrimage journey highlights its group dimension, a crucial factor of the individual religious experience. Using the tools of discourse analysis, argumentation in discourse and the semiotics of the image, this research reveals different forms of collective identity constructed in the discourse of tourism: a particular form of "national" identity is formulated in the speech that Jürgen Bühler, president of the International Christian Embassy in Jerusalem, addresses to African viewers of the Evangelist television channel (LMTV) from his studios in Ivory Coast; a group identity based on the social values of friendship and solidarity is reflected in the 
advertising brochures that the Gota travel agency distributes in Benin Christian communities; finally, the formation of an elite group is realized through the symbolic value of the material objects - certificates and decorations - that the pilgrims accumulate during and after the trip. This research on the discourse and representations of the African pilgrimage to the Holy Land highlights its social dimension; it shows that it goes far beyond the spiritual experience of the isolated believer in search of God.

Cet article porte sur un phénomène culturel peu connu des chercheurs qui s'intéressent au tourisme religieux en Terre Sainte : les pèlerinages effectués à partir de l'Afrique francophone subsaharienne. Cette étude du voyage de pélerinage met en évidence sa dimension groupale, facteur crucial de l'expérience religieuse individuelle. À l'aide des outils de l'analyse du discours, de l'argumentation dans le discours et de la sémiotique de l'image, cette enquête dévoile différentes formes d'identité collective qui se construisent dans le discours du tourisme tel qu'il se développe dans l'univers francophone africain. Une forme particulière d'identité "nationale » est forgée dans le discours que Jürgen Bühler, le président de l'Ambassade Chrétienne internationale de Jérusalem, adresse aux téléspectateurs africains de la chaîne de télévision évangéliste (LMTV) depuis ses studios en Côte d'Ivoire; une identité groupale fondée sur les valeurs sociales de l'amitié émerge dans les affiches publicitaires que l'agence de voyage Gota distribue dans les communautés chrétiennes béninoises; enfin, la formation d'un groupe d'élite se constitue à partir de la valeur symbolique des objets matériels - attestations et décorations que les pèlerins accumulent durant et après le voyage. Cette analyse des discours et représentations du pèlerinage en Terre Sainte met en évidence sa dimension sociale : il apparaît qu'il va bien au-delà de l'expérience spirituelle du croyant isolé à la recherche de Dieu.

\section{INDEX}

Mots-clés: Afrique, évangélisme sioniste, discours publicitaire, identité collective, pèlerinage en Terre Sainte, tourisme religieux

Keywords: Africa, collective identity, Evangelical Zionism, Pilgrimage to the Holy Land, publicity discourse, religious tourism

\section{AUTHOR}

\section{TAL SELA}

Université Bar-Ilan, ADARR 